\title{
Superconducting circuit simulator of Bose-Hubbard model with a flat band
}

\author{
Xiu-Hao Deng, Chen-Yen Lai. ${ }^{*}$ and Chih-Chun Chien \\ School of Natural Sciences, University of California, Merced, CA 95343, USA.
}

\begin{abstract}
Recent advance in quantum simulations of interacting photons using superconducting circuits offers opportunities for investigating the Bose-Hubbard model in various geometries with hopping coefficients and self-interactions tuned to both signs. Here we investigate phenomena related to localized states associated with a flat-band supported by the saw-tooth geometry. A localizationdelocalization transition emerges in the non-interacting regime as the sign of hopping coefficient is changed. In the presence of interactions, patterns of localized states approach a uniform density distribution for repulsive interactions while interesting localized density patterns can arise in strongly attractive regime. The density patterns indicate the underlying inhomogeneity of the simulator. Two-particle correlations can further distinguish the nature of the localized states in attractive and repulsive interaction regimes. We also survey possible experimental implementations of the simulator.
\end{abstract}

PACS numbers: 42.50.Pq 05.30.Jp 74.81.Fa 64.60.an

\section{INTRODUCTION}

Over the past decade intensive research has been focused on studying strongly correlated states of interacting photons in lattices using various quantum systems as simulators1 7 . Among various simulation schemes, superconducting quantum circuits have became a particularly promising platform with a close relation to quantum computation 3 [/46. Many creative ways to simulate quantum systems with superconducting circuits have been proposed or implemented. For instance, a variational optimization over continuous matrix product states has been implemented by using an open circuit quantum electrodynamics (QED) system to simulate the ground state of the Lieb-Liniger mode ${ }^{89}$. Moreover, a digital quantum simulator can be realized using qubits in a digital quantum computation platform, where the corresponding quantum operators of the simulated system are encoded in the Pauli operators of single qubits along with a series of one- or two-qubit gates. A simulator for the Fermi Hubbard model has been demonstrated using a programmable X-mon array and a simulator of the BoseHubbard model using a similar system has been proposed as well 10 .

On the other hand, an analog quantum simulator can provide an intuitive description of many-body systems. One class of simulators can be built with an array of superconducting circuit elements usually fabricated on a chip 3 [4|4|11]. The quanta of the excitations on those circuit elements simulate an ensemble of quantum particles. For example, coupled superconducting qubits as an analogue of a spin array can be a simulator of the Ising mode ${ }^{12] 13}$. Alternatively, photonic excitations in a circuit-QED array may serve as an analogue of lattice bosons 14 , 16 , and effective photon interactions could be created by utilizing strong light-matter couplings between superconducting resonators and qubits $3 / 4|14| 16$. By fabricating circuit QED elements in desired patterns, various lattice structures can be explored and local controls over coherent or dissipative dynamics can also be studied $\frac{6 \mid 15+20 !}{20}$. The latter scheme further allows for simulations of photonic or polaritonic Bose Hubbard model (BHM) 6 6117.

The BHM has been an important paradigm in manybody theories 2122, in particular the Mott insulatorsuperfluid (MI-SF) phase transition it describes. The Mott insulator is a localized state occurring at integer fillings when interaction energy dominates, while the superfluid is a delocalized state where kinetic energy dominates. In superlattices or other complex geometries, the BHM can exhibit many interesting phases and phenomend 237. In certain geometries with multiple sites per unit cell, some of the bands known as flat bands can become non-dispersive. Particles in a flat band form degenerate localized eigenstates, and this particular feature may lead to interesting phases including supersolid ${ }^{28}$ or topological insulator $\stackrel{29}{ }$. The degeneracy of a flat band, on the other hand, is very sensitive to external perturbations and can be lifted easily. The phase diagram of the BHM is enriched if the system supports a flat band, which can be constructed in several known geometries 23/29 31 . A number of constructions of flat-band lattices using graph theory also have been suggested 32 34. Some of the geometries supporting flat bands can be realized in quantum simulators such as optical lattices for cold atoms or photonic crystals using micro cavities ${ }^{35-38}$, although having broadly tunable parameters and periodic boundary conditions remains a great challenge.

Motivated by great opportunities from superconducting circuit simulators, we investigate the BHM on the saw-tooth lattice using an array of superconducting circuit elements with tunable couplers. From the analysis of a superconducting circuit simulator of the BHM outlined in Ref. 17, the simulator in the dispersive regime has a widely tunable parameter range according to its architecture. The superfluid (SF), Mott insulator (MI), and the MI-SF transition may be demonstrated and manipulated 17 . A recent experiment has shown possibilities of simulating attractive bosons modeled by the BHM using an array of transmons 39 . Due to intrinsic anharmonicity of transmons, it is challenging for the simulator of Ref. 39 to exhibit a MI-SF transition or investigate the repulsive regime. A simulator capable of exploring the BHM with 
attractive as well as repulsive interactions, positive as well as negative hopping coefficients, and flexible geometry allowing for a flat band ${ }^{30}$ will elucidate rich physics of the BHM. In the following we will outline a simulator based on Ref. 17 that can simulate non-interacting photons and photons with repulsive or attractive onsite interactions with positive or negative photon hopping coefficients. Interesting localization phenomena associated with flat bands in selected geometries and energy competitions in strongly interacting regimes can be demonstrated by the simulator with available experimental parameters. Moreover, the interesting localization and delocalization phenomena survive in the presence of small fluctuations of system parameters.

This paper is organized as follows. Sec. III introduces the Bose Hubbard model on the saw-tooth lattice and flat-band physics in the thermodynamic limit. In Sec. [III, we review the superconducting circuit simulator for the BHM on periodic saw-tooth lattices and its experimental parameter range. Sec. III presents a discussion on a localization-delocalization transition due to the presence of a flat-band and its experimental signatures. Moreover, different ground states in the repulsive and attractive regimes can be distinguished by two-particle correlation functions. Relevant issues on experimental realizations of the simulator are also discussed. Finally, Sec. V concludes our work.

\section{BOSE HUBBARD MODEL ON SAW-TOOTH LATTICE}

In the infinite saw-tooth lattice shown in Fig. 1(a) with a particular ratio of hopping coefficients, a flat band is separated from the other dispersive band as shown in Fig. 1(c). Whether the flat band is the lowest or the highest energy band depends on the sign of hopping coefficients, and interesting phases can arise due to the flat band. A quantum simulator using superconducting circuits, capable of demonstrating site-wise manipulations of the BHM ${ }^{17}$, is implemented here to demonstrate signatures of the flat band structure (FBS). In the following we consider realistic experimental parameters. Localized states in the flat band are very sensitive to inhomogeneity of the system, and we will show that the patterns of density distributions can be used as a probe of imperfections of the simulator.

In the tight-binding approximation, the BHM Hamiltonian on the saw-tooth lattice is

$$
\begin{aligned}
\mathcal{H}_{0} & =-t_{1} \sum_{\langle i j\rangle}\left(a_{i}^{\dagger} a_{j}+a_{j}^{\dagger} a_{i}\right)-t_{2} \sum_{\langle i j\rangle}\left(a_{i}^{\dagger} b_{j}+b_{j}^{\dagger} a_{i}\right) \\
& +\sum_{i} U_{i} a_{i}^{\dagger} a_{i}\left(a_{i}^{\dagger} a_{i}-1\right)+\sum_{i} U_{i} b_{i}^{\dagger} b_{i}\left(b_{i}^{\dagger} b_{i}-1\right) \\
& +\sum_{i} \mu_{a i} a_{i}^{\dagger} a_{i}+\sum_{i} \mu_{b i} b_{i}^{\dagger} b_{i}
\end{aligned}
$$

where $a_{i}\left(a_{i}^{\dagger}\right)$ and $b_{i}\left(b_{i}^{\dagger}\right)$ are the annihilation (creation) operators on the sub-lattice $A$ and $B$ shown in Fig. 1(a).
To simplify the discussion, we choose $\mu_{i}=0$ across the lattice. When $t_{2}=\sqrt{2}\left|t_{1}\right|$ and $U=0$, the lattice supports a flat band $\sqrt{30}$, which is the lowest-energy band if $t_{1}<0$. The Hamiltonian has the energy spectrum

$$
E\left(k_{x}\right)=2 t_{1} \text { and }-2 t_{1}\left(1+\cos k_{x} a\right)
$$

depicted in Fig. 1(c) with a negative value of $t_{1}$. Here $a$ is the lattice constant and will serve as the length unit. In a finite periodic lattice, the flat band appears as a set of degenerate localized states that are eigenstates of the Hamiltonian. Due to a lack of kinetic energy, flatband states do not participate in transport. In contrast to the Mott insulator existing only at integer fillings, the flat-band states are due to the underlying geometry and could be understood from a single-particle picture. To make connections with realistic superconducting circuit simulators consisting of finite numbers of elements $\frac{17}{4}$, we consider a periodic lattice, for example the one with three unit cells shown in Fig. 11(b).

One important feature of the simulator discussed here is that the hopping coefficients of bosons can be tuned to positive $\left(t_{1}>0\right)$ or negative $\left(t_{1}<0\right)$ values. When there is no interaction, the flat band is the lowest energy band if $t_{1}<0$, so in the ground state the system favors localized states in the flat band. On the other hand, if $t_{1}>0$, the lowest-energy band is dispersive and a uniform groundstate density distribution from delocalized states is expected. Therefore, a localization-delocalization transition occurs as $t_{1}$ changes sign, which could be realized and observed in the proposed simulator by tuning the coupler connecting adjacent lattice sites.

\section{SUPERCONDUCTING CIRCUIT SIMULATOR}

Here we briefly review the superconducting circuit simulator and details can be found in Ref.17. Fig.11(d)-(e) illustrate the elements and their couplings in the simulator, which utilizes an array of superconducting transmission line resonators (TLRs) representing the sites in the BHM. Microwave photons in the TLRs will simulate the bosons in the BHM ${ }^{17}$. Adjacent sites are connected via superconducting quantum interference devices (SQUIDs). In addition, each TLR is capacitively coupled to a tunable charge qubit with a SQUID-like structure ${ }^{\sqrt{17 / 40}}$, which can be used to tune the effective on-site interaction of the BHM.

The Hamiltonian of the simulator consists of on-site terms and coupling terms

$$
H=\sum_{i} \mathcal{H}_{i}^{\text {site }}+\sum_{\langle i j\rangle} \mathcal{V}_{i j}^{\text {couple }}
$$

Here $\langle i j\rangle$ denotes neighboring pairs as shown in Fig. 11(b). The on-site term, $\mathcal{H}_{i}^{\text {site }}$, models a combination of one TLR and a superconducting charge qubit coupled via a capacitor. In Fig. 1 (d) and (e), a TLR is represented by a thick horizontal line, the charge qubit is shown as the 

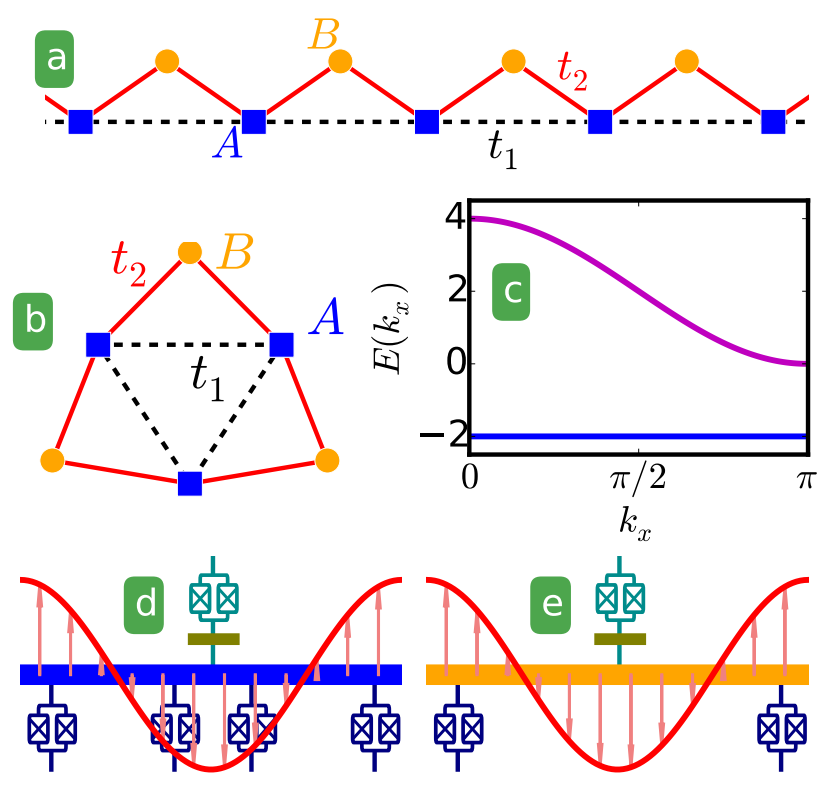

FIG. 1. (Color online) (a) A segment of the saw-tooth lattice. (b) A periodic saw tooth lattice. Here the squares (circles) denote the A-sites (B-sites), and the solid (dashed) lines denote the A-B (A-A) links. (c) Tight-binding bands of the saw-tooth lattice in the thermodynamic limit with a negative tunneling coefficient. (d) The elements forming the A-site in (b). (e) The elements forming the B-site in (b). In (d) and (e), the thick horizontal lines denote the TLRs and the couplers are made of SQUIDs (with two Josephson junctions in each loop). Each A-site is connected to four neighbors by the SQUIDs coupled to the positions depicted in (d). Each B-site is connected to two neighbors as depicted in (e). The sinusoidal curves illustrate the fundamental modes in the TLRs. The charge qubits correspond to the SQUIDs above the TLRs in (d) and (e), and they are coupled to the TLRs via capacitors (the short horizontal lines above the TLRs) to tune the effective on-site photon-photon interaction.

SQUID above the TLR, and the capacitor is denoted by a short line in between them. Details of the modeling are given in Appendix. A.

A deeply off-resonant qubit in the dispersive regime coupled to a TLR gives rise to the following Hamiltonian with an effective on-site photon-photon interaction ${ }^{17}$

$$
\mathcal{H}_{i}^{\text {site }}=\sum_{i}\left[\omega_{i}^{*} c_{i}^{\dagger} c_{i}+U_{i}^{p} c_{i}^{\dagger} c_{i}\left(c_{i}^{\dagger} c_{i}-1\right)\right] .
$$

Here $\omega_{i}^{*}$ is the dressed TLR frequency 14 . With rotating wave approximation and dispersive condition, the on-site repulsion strength $U_{i}^{p}$ can be controlled by qubit-TLR coupling and detuning as explained in Appendix. A. By adjusting the detuning between the qubit and TLR, $U_{i}^{p}$ can be positive or negative.

The coupling term $\mathcal{V}_{i j}^{\text {couple }}$ models a coupler SQUID consisting of two Josephson junctions, which gives rise to a sum of a fixed capacitive coupling and a tunable inductive coupling between neighboring sites ${ }^{17 / 41 / 42}$. Explicitly,

$$
\mathcal{V}_{i j}^{\text {couple }}=-\left(g^{\text {cap }}+g^{\text {ind }}\right)\left(c_{i}^{\dagger} c_{j}+c_{i} c_{j}^{\dagger}\right)
$$

where $g^{\text {cap }}\left(g^{\text {ind }}\right)$ is the capacitive (inductive) coupling constant across the link $i j$. Here we assume all the SQUID couplers are identically fabricated. As shown in Fig.1 (d), the two coupler SQUIDs below the middle of the TLR are placed at $3 / 8$ and $5 / 8$ of the TLR, which fine tune the ratio of the hopping coefficients between the A-A and A-B links in the saw-tooth lattice. Furthermore, the inductive coupling constant, $g^{i n d}$, can be tuned to positive or negative values by changing the magnetic flux through the SQUID.

Here, we limit $\mathcal{V}_{i j}^{\text {couple }}$ to the weak coupling regime and keep only the lowest order when modeling the onsite interaction of Eq. (1). The total Hamiltonian now has the Bose-Hubbard form

$$
\begin{aligned}
H & =\sum_{i}\left[\omega_{i}^{*} c_{i}^{\dagger} c_{i}+U_{i} c_{i}^{\dagger} c_{i}^{\dagger}\left(c_{i} c_{i}-1\right)\right] \\
& -\sum_{<i j>}\left(g^{c a p}+g^{i n d}\right)\left(c_{i}^{\dagger} c_{j}+c_{i} c_{j}^{\dagger}\right) .
\end{aligned}
$$

Compared to Eq. (1), we can construct a mapping between this circuit model and BHM where hopping coefficient $t_{i}=g^{c a p}+g^{i n d}$, onsite energy $\mu_{i}=\omega_{i}^{*}$, and onsite coupling constant $U_{i}=U_{i}^{p}$. Different values of $t_{1}$ and $t_{2}$ can be obtained by adjusting $g^{\text {ind }}$ and both signs of hopping coefficients can be achieved by using typical experimental data summarized in Appendix A. This feature makes the simulator particularly suitable for studying flat-band induced phenomena because by changing the sign of $t_{i}$, relative orders of the energy bands can be reversed. We remark that this circuit model is derived in the deep dispersive regime, where the on-site qubit is not excited due to a large detuning. The only on-site excitation quanta are resonant photons behaving like bosons in the TLR. Therefore, Eq. (6) describes the photonic BHM.

By consulting available experimental data (summarized in Appendix A), in the following we estimate $t_{i}$ in the range of $-10 \mathrm{MHz}$ to $10 \mathrm{MHz}$ and sample three regimes in the phase diagram of interacting photons using the superconducting circuit simulator with uniform $U_{i}=U:$ (a) $U \in[-5,-0.1] \mathrm{MHz}$, (b) $U=0$, and (c) $U \in[0.1,5] \mathrm{MHz}$. For $|U|>5 \mathrm{MHz}$, the qubit-TLR detuning $\Delta_{i}$ may be too small and the on-site excitations could become polariton-like $\frac{43}{45}$. Details of how $\Delta_{i}$ is derived can be found in the Appendix. Although a polaritonic circuit QED lattice may also simulate the BHM with attractive or repulsive effective interactions, the detailed expressions of the on-site energy and interaction are different from the photonic model presented here. The polaritonic system is beyond the scope of our discussion and here we focus on how the photonic simulator can reveal interesting phases in the BHM when a flat band is present. In the photonic simulator, it is difficult 
to approach the $U=0$ point from finite- $U$ because $U$ depends monotonically on the detuning $\Delta_{i}$. Thus, opposite signs of $U$ have to be achieved by starting with opposite signs of the detuning when ground-state behavior is investigated. To access the $U=0$ point, one may detach the qubit from the TLR and completely shut down the onsite interaction (see Appendix A for more details). Figure 3 (a) shows finite- $U$ regimes and the noninteracting regime accessible by the photonic simulator.

\section{A. Inhomogeneity of the simulator}

A realistic simulator will inevitably have imperfections from its fabrication and operation. As a consequence, $\omega_{i}^{*}$ and $t_{i}$ in each sample will fluctuate rather than being perfectly uniform. In addition, quantum fluctuations in Josephson junctions can further contribute to imperfections of superconducting circuit simulators. Here we assume variations of $\omega_{i}^{*}$ and $t_{i}$ due to unwanted crosstalks between the TLRs can be minimized by carefully designing the chip shielded from external devices. The fluctuations of $\omega_{i}^{*}$ in different TLRs are estimated as $\delta \omega / \omega=\left\langle\left|\omega_{i}^{*}-\omega_{j}^{*}\right| / \omega_{i}^{*}\right\rangle \sim 0.1 \%$ based on the following analysis. From available experimental data showing typical TLR frequencies accurate up to $10^{-3} \mathrm{GHz}$ even in a multi-resonator system $[39 \sqrt{46}$, we estimate that the inaccuracy of resonator frequency is around $0.1 \%$ considering the typical value of the resonant frequency $\sim 10$ GHz. The high quality factors $Q>10^{4}$ of TLRs 47 and photon life time up to milli-seconds 48 ensure that quantum fluctuations of $\omega_{i}$ are much smaller than fluctuations from fabrications. Thus, the uncertainties are mainly due to geometrical variation of the TLRs, which is around $\delta l / l \sim 0.1 \%$ 49. The inaccuracy of resonator frequency due to variation of the TLR width can be minimized to about $10^{-4} \mathrm{GHz}^{50}$. Hence, the variance of resonant frequency $\omega^{r}=\frac{2 \pi}{\sqrt{C^{r} L^{r}}}$ due to non-uniformity of the resonator length, which mainly affects the capacitance $C^{r}$, is approximately $\delta \omega / \omega \propto \delta l / l \sim 0.1 \%$. Furthermore, the dressed frequency $\omega^{*}$ of the TLRs can be finely adjusted by tuning the qubit energy ${ }^{14}$, which indicates a feasible way to calibrate the on-site energy $\mu_{i}$. This leads to a even smaller variance of $\delta \omega / \omega$.

For the Josephson junctions in the simulator, there can be more uncertainties in their fabrications leading to larger deviations of the critical current and effective capacitance, which in turn cause variation of $t_{i}$ to be about $1 \% 5154$. Another source of stochastic fluctuations is the magnetic flux noise through a SQUID exhibiting a typical $1 / f$ power spectrum in the range of $1 \sim 10 \mathrm{~Hz}$ 55. The flux noise gives rise to variation of the Josephson energy of SQUIDs and leads to variations of hopping coefficients $t_{i}$ on the order of $10 \mathrm{kHz} 2$. Inhomogeneity of $t_{i}$ due to the noise is estimated as $\delta t / t=\left\langle\left|t_{i}-t_{j}\right| / t_{i}\right\rangle \sim 10 \mathrm{kHz}$ $/ 0.1 \mathrm{GHz}=0.01$. Combining imperfections from fabrication and noise, we estimate $\delta t / t \sim 1 \%$, which is about one order of magnitude larger than $\delta \omega / \omega$. In the following, our numerical simulations will present different ground- state properties assuming fluctuations are dominated by $\delta t$. Although there may also be imperfections in $U$, they do not introduce more physics when $U$ is finite, so $\delta U$ will be neglected.

\section{RESULTS AND DISCUSSIONS}

A flat band can be seen clearly in energy spectrum in the thermodynamic limit shown in Fig. 11(c). However, in a finite system the boundaries will distort the flatband and blur its features. To circumvent finite-size effects, it is desirable to construct a simulator with periodic boundary condition. Fabrication of superconducting circuits gives the simulator considered here some advantages when compared to other possible schemes such as optical lattices ${ }^{35}$ where periodic boundary condition can be difficult. By considering the small number of elements on superconducting chips in current experiments 4|10/46, we start with the smallest periodic saw tooth lattice having only three unit cells as depicted in Fig. 1(b). We choose $\left|t_{2} / t_{1}\right|=\sqrt{2}$ and $\mu_{1,2}=0$, so a flat band is present. In the absence of any imperfection, the flat-band consists of a set of degenerate localized states. The degeneracy, however, is sensitive to imperfections and will be lifted in the presence of tiny inhomogeneity. In a real superconducting simulator, this feature is to our advantage and one can map out the imperfection of a simulator by inspecting spatial patterns of photon distributions.

To account for imperfections of realistic simulators, we include a small fluctuation $\eta=\left|\delta t_{\alpha} / t_{\alpha}\right|$ of the hopping coefficient $t_{1}$ or $t_{2}$. As estimated in section III A fluctuations of the hopping coefficients are about 1\%. In the following we define $t_{\alpha}^{>(<)}=t_{\alpha}(1 \pm \eta)$ and choose $\eta=1 \%$. Imperfections will distort the flat band and favor a particular localized wavefunction as the ground state. Fluctuations of a given simulator are the deviations of its parameters from the averaged values. While fluctuations vary from one sample to another, the magnitude of fluctuations in a given sample may be treated as constant.

\section{A. Single particle picture and noninteracting bosons}

The single particle picture applies when there is only one particle in the system and also to noninteracting bosons in the ground state. By diagonalizing the tightbinding Hamiltonian (1) on a finite size array, the wave function, density distribution, and energy spectrum of the system can be obtained. We show the main result in Fig. 2. As mention previously, the flat band becomes the lowest lying band when $t_{1}<0$. In a finite-size system, the flat band corresponds to several degenerate states. Since any superposition of the degenerate states is still a valid flat-band state, in a perfectly uniform system flatband states could be constructed from superpositions. In contrast, fluctuations of the parameters due to imperfections of the simulator will lift the degeneracy and 
select out a particular ground state. Mapping out the correspondence between the geometry and the pattern of localized states then allows one to visualize features associated with the flat band.

In Fig. 2(a), $t_{1}<0$ and the dominant fluctuation is on a single A-A bond with $t_{1}^{<}=t_{1}(1-\eta)$, which makes the bond weaker (the orange thin line) than the other A-A bonds (in gray). The ground state density distribution is shown, where the sizes of vertices are proportional to the particle density. Particles tend to accumulate on the tip of the triangle with the weaker A-A bond. The reason for this localized state is minimization of kinetic energy in the zero temperature limit. When $t_{1}>0$, the dispersive band becomes the lower-energy band. Then particles will occupy the ground state of the dispersive band, and the density distribution becomes uniform as shown in Fig. 2(a). A localization-delocalization transition of ground states can be observed by comparing density patterns of the two cases with opposite signs of $t_{1}$ while choosing all A-B bonds with $t_{2}=\sqrt{2}\left|t_{1}\right|$.

Next, we consider fluctuations of the two A-B bonds within a triangle with $t_{2}^{>}=t_{2}(1+\eta)$ (the cyan thick lines), which are stronger than the other A-B bonds (in black) as shown in Fig. 2(b). In this case, localized states for $t_{1}<0$ have a similar particle distribution as the case with a weaker A-A bond and delocalized states occur when $t_{1}>0$. The two dual cases have similar density distributions because of similar energy shifts in the triangle with bond fluctuations. We also consider another dual pairs by altering the signs of the fluctuations of the previous two cases. For a stronger A-A bond in Fig. 2(c), the density tends to localize on both ends (blue circles) of the stronger bond (cyan thick line) when the flat band is the lowest band $\left(t_{1}<0\right)$. This case is dual to the case with a pair of weaker A-B bonds (orange thin lines) depicted in Fig. 2(d). By measuring the particle density of a localized state and identifying its pattern, one can infer the location of the dominant imperfection on a simulator.

The single-particle picture is closely related to noninteracting bosons because each boson will occupy the single-particle ground state in the zero-temperature limit. The ground-state density of many bosons is thus the number of particles multiplied by the single-particle ground state density, which can be measured in the superconducting simulator by mapping out the photon number on each site. Thus the ground-state density of a noninteracting bosonic system can be amplified by populating more photons in the system and as a consequence, signatures of the localization-delocalization transition from tuning the signs of hopping coefficients will be more prominent.

\section{B. Interacting Bosons}

When the on-site qubit couples to the TLR in the dispersive regime ${ }^{17 / 44}$, the photons acquire effective on-site interactions and follow the BHM Hamiltonian, Eq. (1). Here we focus on the case of uniform interactions where
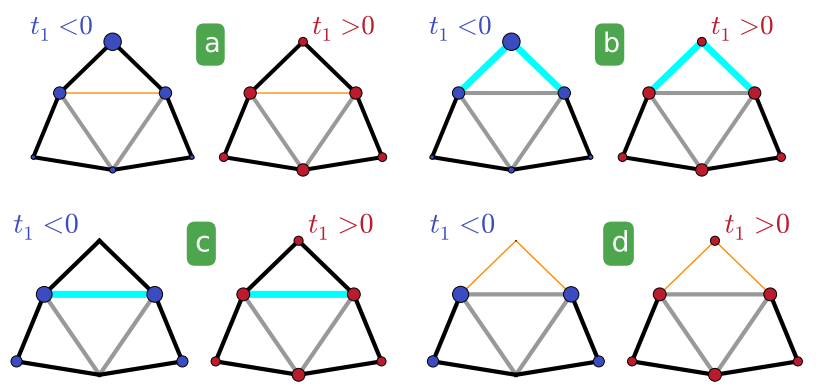

FIG. 2. (Color online) Density distributions of noninteracting particles on periodic saw-tooth lattices with both signs of hopping coefficients, $t_{1}<0$ and $t_{1}>0$. Bigger vertices represent larger particle density, and different bond fluctuation scenarios are compared. Here $\left|t_{2} / t_{1}\right|=\sqrt{2}$. (a) A single weaker $\mathrm{A}-\mathrm{A}$ bond $t_{1}^{<}=t_{1}(1-\eta)$ indicated by the thin orange line. (b) A pair of stronger A-B bonds $t_{2}^{>}=t_{2}(1+\eta)$ marked as the blue thick cyan lines. The density pattern is similar to (a). (c) Single stronger A-A bond $t_{1}^{>}=t_{1}(1+\eta)$ indicated as the thick cyan line. (d) shows the dual case of (c) with a pair of bond fluctuations $t_{2}^{<}=t_{2}(1-\eta)$.

$U_{i}=U, \forall i$. The interaction introduces correlations among the bosons and invalidates the single-particle picture. We therefore use the exact diagonalization (ED) to determine the energy spectrum and ground state wavefunction 56 . The main results are summarized in Fig. 3 , where the system has two interacting bosons in both repulsive $(U>0)$ and attractive $(U<0)$ regimes. Moreover, both positive and negative hopping coefficients $\left(t_{1}>0\right.$ and $\left.t_{1}<0\right)$ are considered. In the noninteracting $(U=0)$ case, a localization-delocalization transition occurs when the sign of the hopping coefficient $t_{1}$ changes. Noninteracting localized states are labeled by the green solid line for $t_{1}<0$ in Fig. 3. As the repulsive interaction increases, we analyze if localized states can be stable against the on-site self-interaction when $t_{1}<0$.

To facilitate a fair comparison with the noninteracting case, two similar sets of fluctuations of the parameters modeling imperfections of the simulator are also included. The first one has a pair of stronger A-B bonds $\left(t_{2}^{>}\right)$and the other has a single stronger A-A bond $\left(t_{1}^{>}\right)$. The former is similar to its dual case with a single weaker A-A bond $\left(t_{1}^{<}\right)$while the latter shows similar localized wavefunctions as the case with a pair of weaker A-B bonds $\left(t_{2}^{<}\right)$. Similarities of particle-density patterns of localized state between dual cases are still observable even in the presence of weak self-interaction.

For repulsive interaction $U>0$, we plot the energy spectra and density distributions for three selected values of the interaction in Fig. 4 including two sets of parameter fluctuations. When $U=0$, the ground state is localized and the density distribution shows localized patterns. As interaction strength increases, the density distribution starts to spread out. In Fig. 4 (a), particles accumulate on the triangle with a pair of stronger A-B bonds when $U=0$. The results coincide with the single 


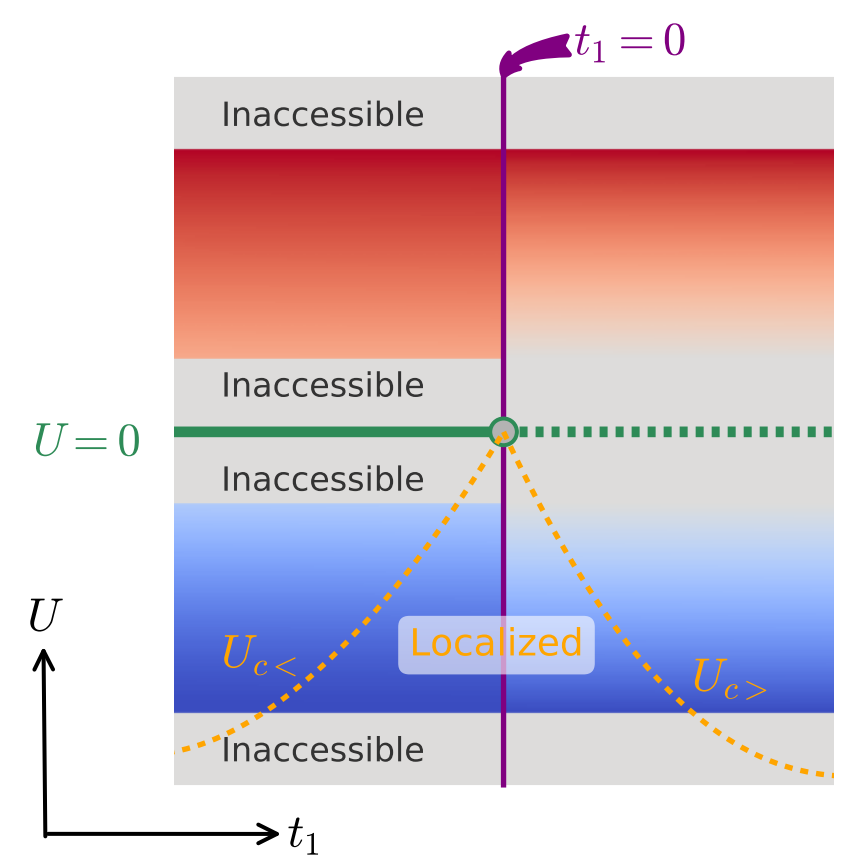

FIG. 3. (Color online) Schematic phase diagram of the proposed superconducting circuit simulator of the BHM with two bosons on a periodic saw-tooth lattice supporting a flat band. Imperfections of the simulator modeled as a weaker A-A bond $t_{1}^{<}=t_{1}(1-\eta)$ have been included. The gray areas indicated as inaccessible are beyond the validity of the simulator. The green solid line indicates localized states associated with the flat band, and the green dashed line indicates delocalized states. In the regime labeled "Localized" below the dashed yellow lines, strong attractive interactions leads to the domination of superpositions of states with all the particles concentrating on each site. For two particles in the system, secondorder processes favor uniformly distributed density, while for more than two particles localized density patterns emerge.

particle picture in this limit. Here localized ground states resulting from the flat-band manifest themselves as degenerate eigenvalues shown in the energy spectrum. We remark that, in the presence of imperfections and interactions, the flat-band is distorted so the ground state can be uniquely determined. As the interaction increases to $U=0.05\left|t_{1}\right|$ depicted in Fig. 4 (c), the density distribution of the ground state spreads out rather than localizing on a single triangle. Before the density distribution becomes completely uniform, particles tend to occupy the triangle on the opposite side of the strongest fluctuation, which will be discussed later in the context of a larger system.

Finally, when the interaction strength reaches the same order as the hopping coefficient, $U=\left|t_{1}\right|$ in Fig. 4(e), the particle distribution becomes uniform and the flat-band spectrum no longer exists because localized states are not eigenstates of the full Hamiltonian when the interaction is strong. Similar phenomena are also discovered in the other case shown in Fig. 4(b), (d), (f), where the system has only one single stronger A-A bond. In the presence of strong self-interactions, the ground state exhibits uni- formly distributed particle density.

Similarly, the spreading of the density distribution can be observed when the interaction is attractive. From the real-space density distribution, one cannot discern the difference between the results from intermediate attractive and repulsive interactions because both cases show almost uniform distributions. However, a two-particle correlation called the density correlation across the link (DCAL) can distinguish features of the two interaction regimes. The DCAL is defined as

$$
N_{l i n k}=\sum_{<i j>}\left\langle\hat{n}_{i} \hat{n}_{j}\right\rangle
$$

Here the summation only includes the pairs of sites across links, $\hat{n}_{i}$ is the particle number operator on site $i$, and $\langle\cdots\rangle$ denotes ground-state expectation value. In the repulsive regime, the DCAL varies slowly with the interaction and reaches a finite steady value in the strongly interacting regime. On the other hand, the DCAL in the attractive regime keeps decreasing. Moreover, the DCAL vanishes when the attraction exceeds a critical value, $|U|>\left|U_{c<}\right|$. This indicates the dominance of superpositions of doubly occupied states taking the form $\Psi=\sum_{i} u_{i}\left|0, \cdots, n_{i}=2, \cdots, 0\right\rangle$, where the Fock states label the particle number on each site. In this case, $N_{l i n k}=0$. It has been proposed that for the attractive BHM in the thermodynamic limit, the difference between states with variable particle numbers per site and states consisting of superpositions of fully occupied sites will become a phase transition $57 / 58$, and our results are in line with the prediction. The yellow dashed lines in Fig. 3 indicate a change of ground state properties in the attractive regime. By measuring the numbers of photons on all sites repeatedly and constructing their products, the DCAL can be extracted.

When more than two particles are loaded into the system, the localization-delocalization transition at $U=0$ is still observable as the hopping coefficient $t_{1}$ changes sign. Fig. 5 shows the case of three bosons on a periodic saw-tooth lattice. The spreading trend of the density at intermediate $U$ is also similar to the two-particle case. A difference between $N=2$ and $N>2$, where $N$ is the total number of bosons in the system, is that localized density patterns emerge again in the strongly attractive regimes $\left(U<0\right.$ and $\left.\left|U / t_{1}\right|>>1\right)$ as shown in Fig. $5(\mathrm{i})$ and $(j)$. The reason for the re-entrance of localized density patterns can be understood from second-order degenerate perturbation theory and the presence of imperfections of the system parameters. Second-order hopping processes select out sectors in the Fock space consisting of states like $|0, \cdots, 0, N, 0, \cdots, 0\rangle$ according to the inhomogeneity of the hopping coefficients, which then cause concentration or distillation of the density in the region with the strongest fluctuations. Interestingly, when there are only two bosons in the system, the selection process favors uniform density distribution. Moreover, the localized density patterns in the strongly attractive regime for $N>2$ can be observed with negative as well as positive hopping coefficients, but the localized density patterns 

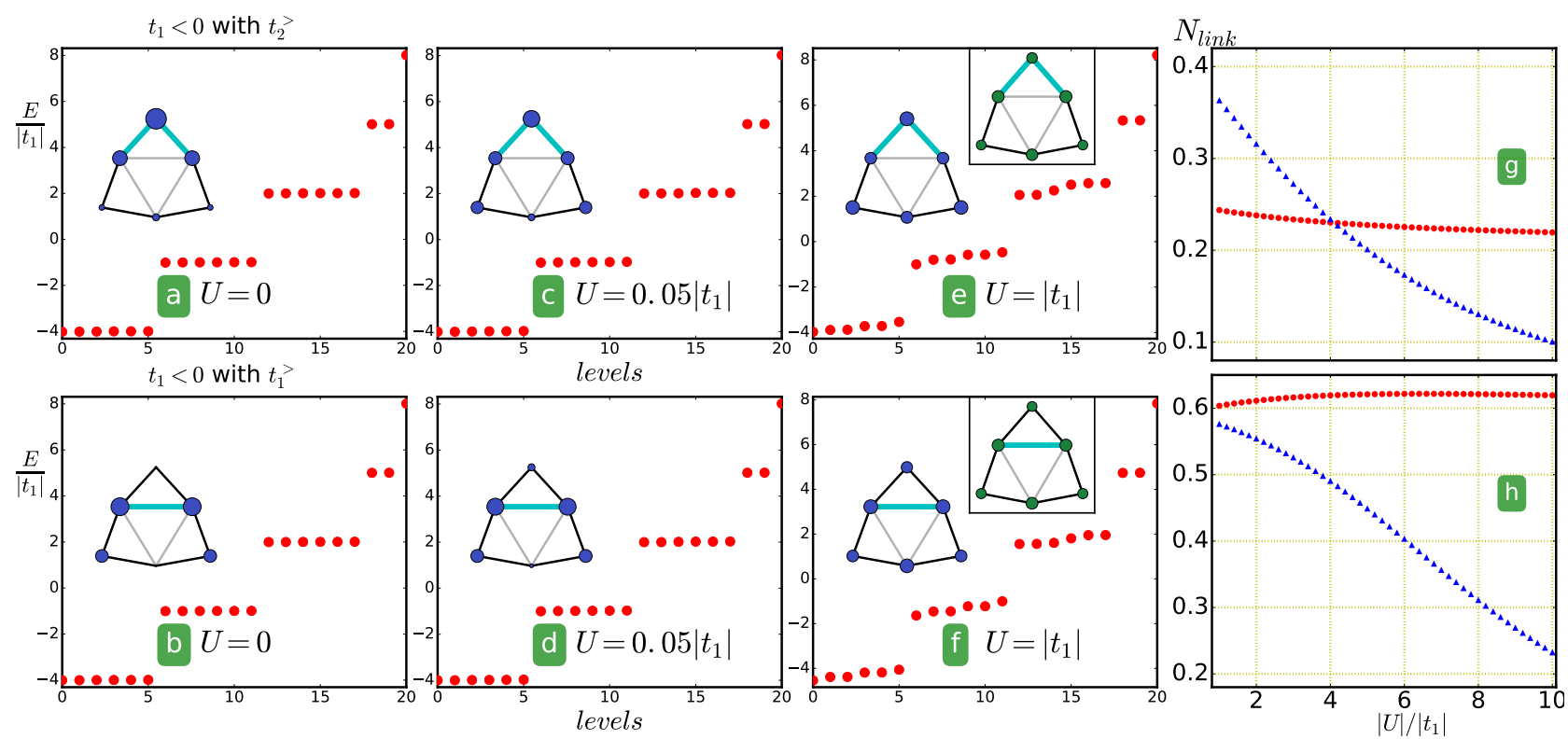

FIG. 4. (Color online) Energy levels and ground state density patterns (inset) of two bosons with $t_{1}<0$ and different interaction strength. Here $\left|t_{2} / t_{1}\right|=\sqrt{2}$. The left column shows a system with a pair of bond fluctuations $t_{2}^{>}$indicated by the thick cyan lines in the insets. The right column shows a system with one bond fluctuation $t_{1}^{>}$indicated by the thick cyan line in the insets. A bigger vertex indicates a larger density in each inset. The non-interacting cases, (a) and (b), show nearly degenerate low energy states. Imperfections of the simulator lift the degeneracy of low-energy eigenstates and single out a unique ground state. When $U / t_{1}$ is small, splitting of low-energy levels is tiny and not discernible with the resolution shown here. For a small repulsive interaction $U=0.05\left|t_{1}\right|$ in (c) and (d), we still see localized ground state density patterns, and low-energy levels stay nearly degenerate. As the interaction gets stronger $\left(U=\left|t_{1}\right|\right.$ in (e) and (f)), density patterns become uniformly distributed and low-energy levels are dispersive. The insets in (e) and (f) inside the square frames show the density patterns of the attractive cases with $U=-\left|t_{1}\right|$ and same imperfections. The density distributions are similar. (g) DCAL $N_{\text {link }}$ (defined in Eq. (7)) for $t_{1}>0$. (h) DCAL for $t_{1}<0$.

are different when the hopping coefficients change sign. A more rigorous analysis of a three-site system is given in Appendix B. No re-entrance of localized density patterns are observed in the repulsive regime for $N=2,3$ and this should also apply to $N>3$.

The DCAL in the strongly repulsive regime saturates to a value depending on the total particle number, but in the attractive case the DCAL always decays toward zero as shown in Fig. 5 (k) and (l). Therefore, the DCAL reveals the subtle difference between states in the attractive and repulsive regimes accessible by the superconducting circuit simulator with or without inhomogeneity in the parameters. We remark that weakening of localized states by interactions in the BHM has been proposed in Refs. 21 and 23.

\section{Larger arrays of the simulator}

By considering systems with four, five, and six unit cells of the saw-tooth lattice, we can further address the behavior of localized states. The density distributions of three bosons without interaction $(U=0)$ and hopping coefficient $t_{1}<0$ are shown in Fig. 6(a)-(c) with different numbers of unit cells respectively. As mentioned before, localized states are favorable on the triangle with the strongest bond fluctuations due to imperfections of the simulator. Fluctuations of the hopping coefficient with realistic parameters can be controlled to be within $1 \%$.

In the weakly repulsive regime, for instance $U=0.1\left|t_{1}\right|$ shown in Fig. 6(d)-(f), the particles tend to occupy the opposite side of the triangle with the most fluctuations in order to stay away from each other. This tendency avoids the on-site self-interaction already at the zeroth order. On the other hand, for the attractive cases shown in Fig. 6(g)-(i) we also see spreading of localized states with $U=-0.5\left|t_{1}\right|$. Although the density patterns in both strongly attractive and repulsive regimes are similar, the trends are different. In the attractive regime the density starts to populate the region near the triangle with the largest bond fluctuations rather than occupying the opposite triangle in the repulsive cases.

As the repulsive interaction gets stronger, for example $U=10\left|t_{1}\right|$ shown in Fig. 6(j)-(l), the density distributions eventually become uniform. On the other hand, strongly attractive interaction drives the system with $N>2$ into another regime with localized density patterns as shown in Fig. 6(m)-(o) with $U=-10\left|t_{1}\right|$. For $N=2$, the density distribution remains uniform in the strongly attractive regime. The strongly attractive regime with $t_{1}>0$ or $t_{1}<0$ are labeled as "Localized" in Fig. 3 to emphasize the emergence of localized density patterns when 

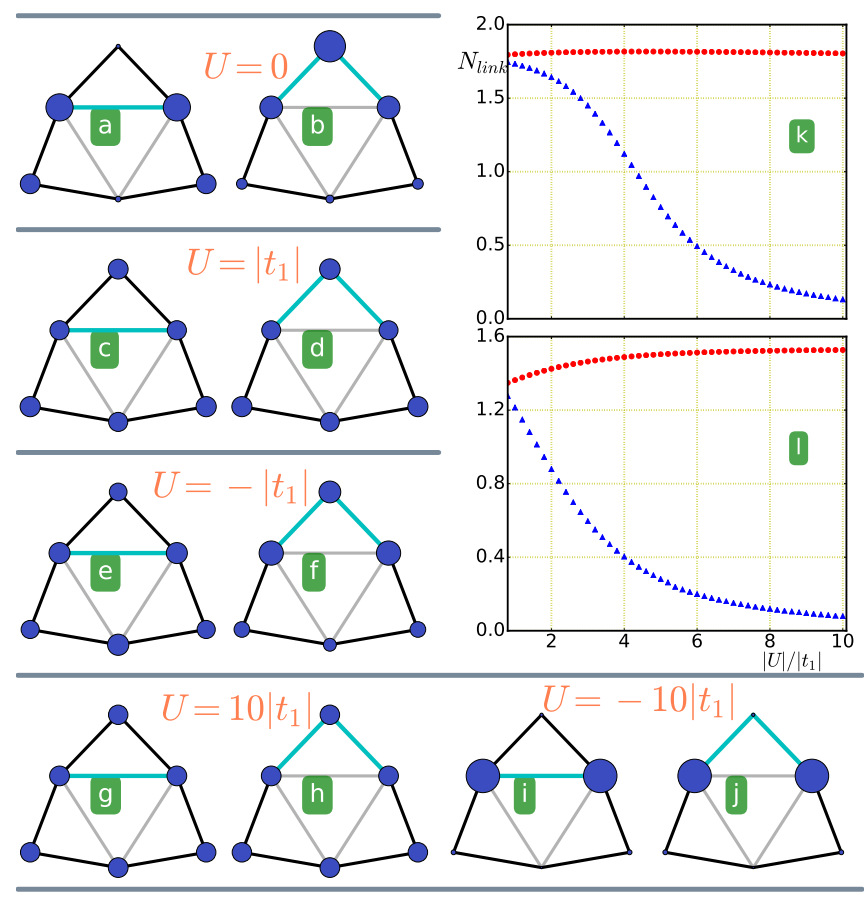

FIG. 5. (Color online) Density distributions of three bosons on a periodic saw-tooth lattice. (a)-(b) Noninteracting cases, (c)-(d) weakly repulsive cases, (e)-(f) weakly attractive cases, (g)-(h) strongly repulsive cases, and (i)-(j) strongly attractive cases. Here $t_{1}<0$ with bond fluctuations (cyan lines) $t_{1}^{>}$on a single A-A bond (left column) and $t_{2}^{>}$on a pair of A-B bonds (right column). $\left|t_{2} / t_{1}\right|=\sqrt{2}$. The localized states in the noninteracting cases have the same structure as the case with two particles. In the repulsive regime, the density patterns gradually become uniformly distributed. In contrast, another regime with localized density patterns emerges in the strongly attractive regimes. The DCAL $N_{\text {link }}$ (defined in Eq. (7) for $t_{1}>0$ and $t_{1}<0$ are shown in (k) and (1), respectively. The repulsive case (red circles) and attractive case (blue triangles) approach different values in the strongly interacting regimes.

more than two interacting bosons are present.

\section{Implications for experimental implementations}

Before closing our discussion, we briefly comment on experimental realizations of the simulator.

State preparation: Innovative ways have been investigated for preparing a deterministic product Fock state in multi-TLR systems 4 446/50. For instance, in Ref. 17 the TLRs in the simulator (the on-site TLR) may be connected to auxiliary control TLRs via additional tunable couplers which can be formed by several SQUIDs. It has been assumed that one can generate any numbers of photon in the control TLR ${ }^{54}$. By adjusting the qubit energy and sending in pump signals to compensate for the detuning between the on-site TLR and the control TLR, one can swap the photon states between the on-site TLR and control TLR ${ }^{59}$ and prepare a deterministic Fock state in

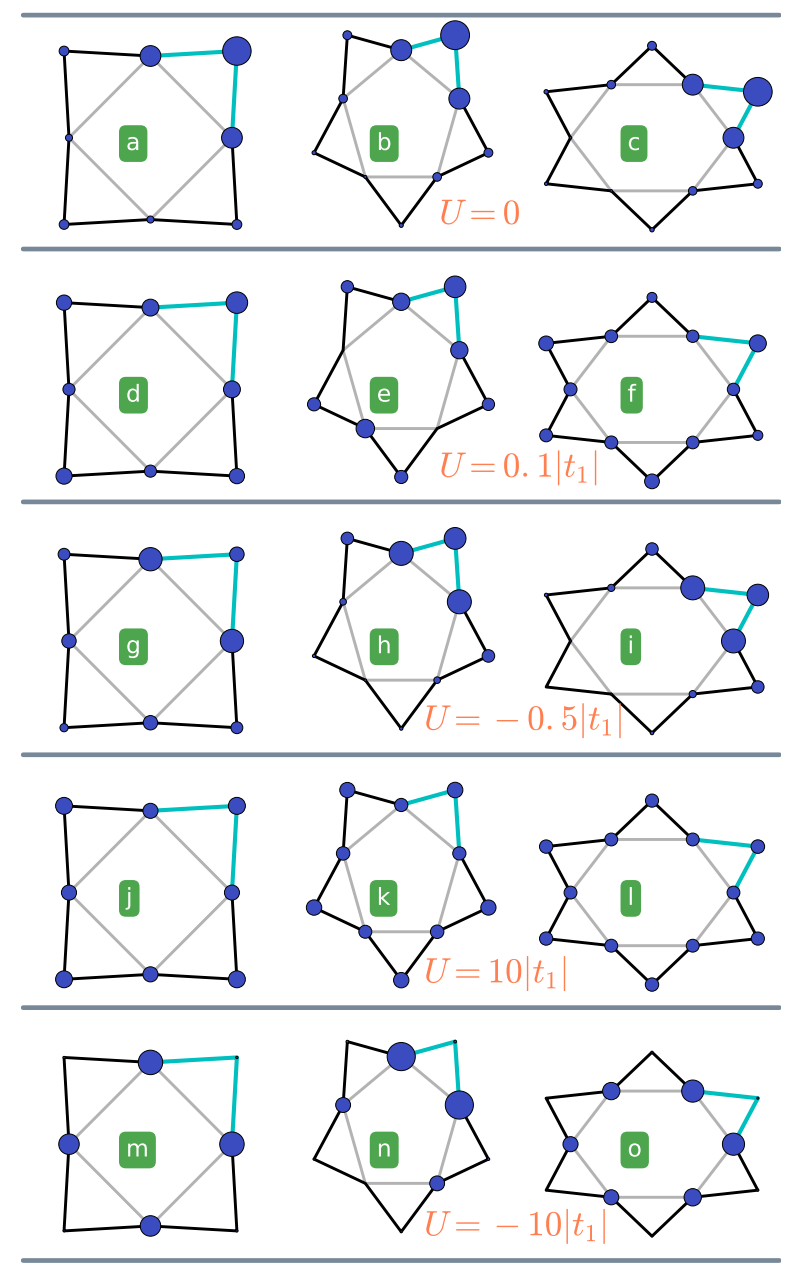

FIG. 6. (Color online) Density distributions of three bosons on different lattices: Four unit cells in the left column, five unit cells in the middle column, and six unit cells in the right column. Here $t_{1}<0$ with bond fluctuations $t_{2}^{>}$on a pair of A-B bonds (cyan lines) and $\left|t_{2} / t_{1}\right|=\sqrt{2}$. The interaction strength are: (a)-(c) $U=0$ (non-interacting), (d)-(f) $U=0.5\left|t_{1}\right|$ (repulsive), (g)-(i) $U=-0.5\left|t_{1}\right|$ (attractive), (j)-(l) $U=10\left|t_{1}\right|$ (repulsive), (m)-(o) $U=-10\left|t_{1}\right|$ (attractive). Bigger vertices correspond to larger densities. The spreading to the triangle opposite to the triangle with dominant bond fluctuations in the presence of repulsion can be clearly observed by comparing the first and second rows. For attractive interactions, the first and third rows show the spreading occurs near the triangle with dominant bond fluctuations, while the last row shows the re-entrance of localized density patterns. For a system with only two bosons, the density pattern remains uniform in the strongly attractive regime.

the on-site TLR. In this scheme, the numbers of photons on different sites can be different. A product Fock state can be prepared as the initial state of the simulator by applying this process on all the sites simultaneously.

The measurement of the photon number on each site may be performed in a similar way to map out the state in Fock space ${ }^{17}$. Alternatively, quantum non-demolition (QND) measurements may be performed on the on-site 
TLRs so the photon numbers on each TLR could be measured with a weakly-coupled and off-resonant probe signal and the photons in the simulator will not be demolished 60 62.

Cooling: Localized versus delocalized ground states of photons have been contrasted in Figs. 2 to 4 . Once the simulator is prepared in a product Fock state, photons are either localized on certain sites or spreading among different sites. In order to study ground state properties, one needs to cool the system down to the ground state without losing the photons in the system. Hence, a cooling scheme needs: i) Collective excitations of the simulator need to be reduced while conserving the total photon number, which could be achieved by using a cavity-assisted side-band cooling scheme. An experiment has demonstrated a collective ground state of the BHM in the attractive regime simulated by an array of transmons 39 . There have been other theoretical studies 63,65 on number-conserving manipulations of the photon excitations in superconducting circuits providing feasible alternatives. ii) The cooling rate, defined as the sum of the stimulated (pump-assisted) transmission from higher levels to the ground state ${ }^{39}$, of the whole lattice has to be faster than the decay rate of photons. Considering the high Q nature of the TLR, the lifetime of photons can be around milliseconds $s^{47 / 48 \mid 66}$. Thus the cooling rate needs to be in the range of $\mathrm{kHz}$ to $\mathrm{MHz}$.

Tunability of parameters: Broad tunability of the simulator is made possible by the following mechanisms. i) The qubit coupled to the TLR introduces an effective tunable on-site interaction. The repulsive or attractive interaction depends on whether the qubit is red-detuned or blue-detuned from the resonator, and the interaction strength depends on the magnitude of the detuning. ii) The SQUIDs coupling neighbor sites allow the hopping coefficient to be tuned from negative values to positive values. The Josephson junction in a SQUID is modeled as a combination of a capacitor and a tunable nonlinear inductor ${ }^{67}$. When the inductor dominates, the SQUID coupler of the simulator becomes a low pass filter so photons, which are AC electromagnetic signals in the TLR, tend to be blocked by the inductor and yields a positive hopping coefficient. When the capacitor dominates the coupling, the coupler becomes a high pass filter. Therefore, photons tend to hop between different sites and reduce overall energy by hopping, which yields a negative hopping coefficient. Combining those features allows the proposed superconducting system to simulate various phases of the Bose Hubbard model.

\section{CONCLUSION}

Simulations of the BHM on periodic saw-tooth lattices supporting a flat band are feasible by using the versatile superconducting circuit simulator with broadly tunable parameters discussed here. A localization-delocalization transition of noninteracting bosons associated with the flat band of saw-tooth lattices is made possible because the sign of hopping coefficients in the simulator can be controlled. In the presence of onsite interactions, density patterns from localized states are still observable and sensitive to inhomogeneity of the underlying elements. One may exploit this feature and use the density pattern as a diagnosis tool for identifying imperfections.

The rich phase diagram of the BHM with a flat band illustrated in Fig. 3 elucidates interesting interplays between geometry and interaction. Delicate differences between ground states in repulsive and attractive regimes, although not visible in the density distribution, can be discerned by two-particle correlations. Moreover, this work may inspire similar studies in superconducting circuit simulators of fermionic systems $10[68 \mid 69$.

\section{ACKNOWLEDGMENTS}

We thank S. Hacohen-Gourgy and Chunjing Jia for inspiring discussions in the early stage of this work.

\section{Appendix A: Tunability and applicability of superconducting circuit}

Here we summarize the modeling, approximation, and experimental parameters of the superconducting circuit simulator. The on-site term describes a TLR coupled to a superconducting qubit in Eq. (3) as

$$
\mathcal{H}_{i}^{\text {site }}=\omega_{i}^{r} b_{i}^{\dagger} b_{i}+\frac{\omega_{i}^{q}}{2} \sigma_{i}^{z}+g_{i}^{q} \sigma_{i}^{x}\left(b_{i}^{\dagger}+b_{i}\right)
$$

with the TLR frequency $\omega^{r}=\frac{2 \pi}{\sqrt{C^{r} L^{r}}}=2 \pi \sqrt{E_{C}^{r} E_{L}^{r}}$, qubit frequency $\omega^{q}=2 E_{J}^{q} \cos \left(\frac{\phi_{e}}{2}\right)$ when the qubit is capacitively biased at the charge degeneracy point ${ }^{70 / 71}$, and the coupling between the TLR and qubit $g_{i}^{q}=2 e \frac{C_{g}}{C_{\Sigma}^{q}} \sqrt{\omega^{r} C^{r}}$. The Pauli operators $\sigma^{\{x, y, z\}}$ represent the qubit coupled to each TLR. $C^{r}$ and $L^{r}$ are the total capacitance and inductance of the TLR. $E_{C}^{r}=\frac{(2 e)^{2}}{C^{r}}$ and $E_{L}^{r}=\frac{1}{L^{r}(2 e)^{2}}$ are capacitive and inductive energies of the TLR. $E_{J}^{q}$ is the Josephson energy of each junction in the qubit. $C_{g}$ is the coupling capacitance and $C_{\Sigma}^{q}$ is the sum of the total effective capacitance between the TLR and the ground. $\phi_{e}$ is the magnetic flux through the SQUID loop. After the rotating-wave approximation, the on-site interaction coupling constant in Eq. (4) is given by $U_{i}^{p}=g_{i}^{q}\left(\frac{g_{i}^{q}}{\Delta}\right)^{3}$, which can be attractive or repulsive by varying the detuning $\Delta=\omega^{r}-\omega^{q}$ to be negative or positive.

The coupler SQUID in Eq. $5 . \mathcal{V}_{i j}^{\text {couple }}$, contains the capacity coupling constant $g^{c a p}=G_{0} \omega^{r} E_{C}^{j j} / E_{c}^{r}$ and inductive coupling constant $g^{i n d}=4 G_{0} \omega^{r} E_{J}^{j j} \cos \left(\frac{\phi_{e}}{2}\right) / E_{L}^{r}$. $E_{J}^{j j}$ and $E_{C}^{j j}$ are the inductive and capacitive energy of the Josephson junction in the SQUID coupler. The amplitude factor $G_{0}$ depends on where on the TLR the qubit is coupled to. If it is placed at the antinode ${ }^{14}$, the factor $G_{0}=1$. In Fig 1 (d), the two SQUIDs below the middle of the TLR are placed at $3 / 8$ and $5 / 8$ of the TLR, 
hence $G_{0}=\cos (\pi / 4)$ for them. By tuning $\phi_{e}$ in the domain $[0,2 \pi], g^{\text {ind }}$ can be tuned positive or negative. The superconducting circuit model is derived in the large dispersive regime, where $\Delta_{i} \gg g_{i}^{q} \gg t_{i}$.

We now turn to experimental parameters. Available experimental data ${ }^{14} \mid \frac{16|39| 42 / 46|54| 72}{1}$ allow one to set $\omega^{r}=$ $5 G H z, E_{C}^{c}=0.2 G H z, E_{J}^{c}=10 G H z, E_{L}^{r}=50 G H z$, $E_{c}^{r}=0.5 G H z, E_{C}^{c}=0.2 G H z, g^{i n d} \in[-1.2,1.2] G H z$, $g^{c a p}=0.6 G H z$, then the hopping coefficient $t_{i}=g^{c a p}+$ $g^{\text {ind }} \in[-0.3,1.5] G H z$. We consider an appropriate range of $\phi_{e}$, so $t_{i}$ can be tuned in the range $[-0.6,0.6] G H z$. Notice that $g^{c a p}$ needs to be smaller than the tunable inductive term $g^{\text {ind }}$ so the hopping coefficient can be switched between positive and negative values. A small $E_{C}^{c}$ can be achieved by coupling capacitors between the SQUID and TLR. Using only capacitors ${ }^{4}$ or SQUID ${ }^{42}$ coupled to TLRs has been demonstrated in experiments. Here we consider a tunable coupler from a combination of a capacitor and a SQUID in order to access both regimes with positive and negative hopping coefficients. The effective capacitance between two TLRs coupled by a combined coupler can be increased and a low capacitive energy $E_{C}^{c}=(2 e)^{2} / 2 C$ can be achieved.

Similar to the SQUID coupler, a flux bias through the qubit can be used to tune the qubit frequency. In order to apply the simulator to the BHM, additional approximation conditions are imposed: (a) The atomic limit $g_{i}^{q} \gg t_{i}$, where photon hopping can be treated as a perturbation ${ }^{44}$. (b) The dispersive condition $\Delta_{i} \gg g_{i}^{q}$, which decouples the qubit from the TLR and allows a perturbative treatment of the on-site Hamiltonian and the effective on-site interaction $U^{4114144}$. (c) The perturbative condition, which requires the hopping coefficient $t_{i}$ to be about the same order of or smaller than the on-site interaction $g_{i}^{q}\left(\frac{g_{i}^{q}}{\Delta_{i}}\right)^{3}$ as the TLR is coupled to the qubit. Then perturbation theory is applicable ${ }^{17}$.

The capacitive coupling between the qubit and resonator, $g_{i}^{q}$, is fixed once elements are fabricated. Here we take a typical experimental value $g_{i}^{q}=130 \mathrm{MHz}$ 14|54 . In order to keep the simulator in the dispersive regime, the detuning $\left|\Delta_{i}\right|$ should be tuned within $[0.4,5) \mathrm{GHz}_{2} 14 \mid 39$. Therefore, the on-site interaction $U_{i} \in[-5,0) \cup(0,5] \mathrm{MHz}$ and $t_{i}$ can be tuned within $[-10,10] \mathrm{MHz}$. We remark that the on-site coupling constant $U_{i}=g_{i}^{q}\left(\frac{g_{i}^{q}}{\Delta}\right)^{3}$ can be attractive or repulsive depending on whether the qubit is in the red-detuned $(\Delta<0)$ or blue-detuned $(\Delta>0)$ regime. By increasing the detuning $\Delta,\left|U_{i}\right|$ can be reduced. One may expect when the qubit is far off-resonant from the TLR, $U_{i}$ can approach 0 . However, a continuous scanning control of the flux bias used to tune the SQUID frequency does not change the sign of $U_{i}$. Moreover, in the presence of the qubit there is always a non-vanishing value of $U_{i}$. To circumvent difficulties of reducing $U$ towards 0 , one may detach the qubit from the resonator to shut down photon interactions in the resonator completely and reach the limit $U_{i}=0$. Recent experiments ${ }^{42 / 72}$ have shown ultrastrong tunable coupling between two TLRs and they support the estimation of hopping coefficients used here. On the other hand, in the ultrastrong coupling regime, different nonlinear effects $\$ 2172173$ other than simple photon hopping could arise and validity for the BHM simulator breaks down. To explore the phase diagram of the BHM with a flat band, parameters of the simulator should remain in the weak coupling regime, where coupling between neighboring sites (photon hopping) is weaker compared to the on-site qubit-TLR coupling.

\section{Appendix B: Second-order degenerate perturbation in the strongly attractive regime}

According to Appendix A] the hopping coefficients $t_{1,2}$ of the simulator can be continuously tuned around $t_{1,2}=0$, which enables us to study the strongly interaction regime $\left|U / t_{1,2}\right|>>1$ by tuning the ratio. In the strongly attractive regimes, a regime with localized density patterns emerges when the system has more than two particles. Here we use second-order degenerate perturbation theory ${ }^{74}$ to explain the emergence of the regime and why it is only observable for $N>2$. Here we consider the BHM on a three-site lattice forming a triangle. The Hamiltonian is

$$
\begin{aligned}
H & =H_{0}+H_{I} \\
H_{0} & =\frac{U}{2} \sum_{i} n_{i}\left(n_{i}-1\right) \\
H_{I} & =-\sum_{\langle i, j\rangle} t_{i j}\left(b_{i}^{\dagger} b_{j}+\text { h.c. }\right)
\end{aligned}
$$

with onsite coupling constant $U$ and hopping coefficient $t_{i j}$ with $i, j$ denoting the two sites connected by a bond. In the strongly attractive regime with $\left|U / t_{i j}\right|>>1$, we treat $H_{I}$ as a perturbation. When all the hopping coefficients vanish, the unperturbed ground state is any superposition of the three Fock states $|1\rangle=|N, 0,0\rangle$, $|2\rangle=|0, N, 0\rangle$, and $|3\rangle=|0,0, N\rangle$ because of the attractive interaction. Thus, we take the space spanned by the three states and consider corrections due small $t_{i j}$. The unperturbed Hamiltonian is degenerate in the $\{|1\rangle,|2\rangle,|3\rangle\}$ basis and the matrix representation takes the form

$$
H_{0}=\left(\begin{array}{ccc}
E_{N} & 0 & 0 \\
0 & E_{N} & 0 \\
0 & 0 & E_{N}
\end{array}\right)
$$

with $E_{N}=U N(N-1) / 2$. For the three degenerate unperturbed states, all first-order terms vanish because they cannot be connected by exchanging only one particle.

The hopping terms, however, introduce second-order processes with the assistance from higher-energy unperturbed states like $|N-1,1,0\rangle$, etc. For instance, $\Delta E_{11}=$ $\sum_{f}\left\langle N, 0,0\left|H_{I}\right| f\right\rangle\left\langle f\left|H_{I}\right| N, 0,0\right\rangle /\left(E_{f}-E_{N}\right)$, where the intermediate states are $f \in\{|N-1,1,0\rangle,|N-1,0,1\rangle\}$ with energy $E_{f}=U(N-1)(N-2) / 2$. The ground state can 
then be found by diagonalizing the matrix ${ }^{74}$

$$
\left(\begin{array}{ccc}
\Delta E_{11} & \Delta E_{12} & \Delta E_{13} \\
\Delta E_{21} & \Delta E_{22} & \Delta E_{23} \\
\Delta E_{31} & \Delta E_{32} & \Delta E_{33}
\end{array}\right)
$$

In constructing $\Delta E_{i j}$, the unperturbed states are excluded from being used as intermediate states. The diagonal terms $\Delta E_{j j}$ are of the order of $\left|t_{i j}^{2} / U\right|$. The offdiagonal terms, however, are sensitive to the total particle number $N$. When $N=2$, one can see that two degenerate unperturbed states can be connected via an intermediate higher-energy state. For instance, $|2,0,0\rangle$ can hop to $|1,1,0\rangle$ and then to $|0,2,0\rangle$. Therefore, all $\Delta E_{i j}$ are of the order of $\left|t_{i j}^{2} / U\right|$ and the second-order ground state after diagonalizing the matrix is still a superposition of the three unperturbed ground states. As a consequence, the density is mostly uniform.

In contrast, two different unperturbed states cannot be connected via second-order processes when $N>2$. For example, when $N=3$, there is no intermediate state $|f\rangle$ connecting $|3,0,0\rangle$ and $|0,3,0\rangle$ with two hopping events. Therefore, $\Delta E_{i j}=0$ if $i \neq j$ at the second-order level. The diagonal terms, however, are finite at the secondorder level. Moreover, in the presence of fluctuations of the hopping coefficients, $t_{i j}$ are different and this leads to different $\Delta E_{j j}$. Therefore, the matrix (B4) picks up a preferred state in its diagonalization. For example, if $\Delta E_{11}$ is the smallest among $\Delta E_{j j}$, the ground state up to the second order would be $|N, 0,0\rangle$. When $N$ and the number of sites are large, the ground state may remain degenerate up to the second order in a subspace of the original set of unperturbed states, and higher-order perturbations will further lift the degeneracy. The important point is that the ground state, up to the second order, only includes a subset of the unperturbed states, which means the density is concentrated or distilled on certain sites. Therefore, the ground state in the presence of weak hopping coefficients and imperfections of the parameters exhibits localized density patterns when $N \geq 3$.

When there are more than three sites, the second-order degenerate perturbation theory still applies and one expects localized density patterns in the strongly attractive regime when $N>2$. Moreover, the perturbation theory works for both negative as well as positive $t_{i j}$. The localization patterns, though, are different when the sign of $t_{i j}$ changes because higher-order processes sensitive to the sign will further refine the selection of the ground state. When the interaction is repulsive, the ground state always tends to spread out the density and no localization is found in the strongly repulsive regime.
* clai24@ucmerced.edu

1 I. Georgescu, S. Ashhab, and F. Nori, Rev. Mod. Phys. 86, 153 (2014).

2 A. Aspuru-Guzik and P. Walther, Nat. Phys. 8, 285 (2012).

3 M. Devoret and R. Schoelkopf, Science 339, 1169 (2013).

4 A. A. Houck, H. E. Türeci, and J. Koch, Nat. Phys. 8, 292 (2012).

${ }^{5}$ C. Bruder, R. Fazio, and G. Schön, Annalen der Physik 14, 566 (2005).

${ }^{6}$ S. Schmidt and J. Koch, Annalen der Physik 525, 395 (2013).

7 D. Tanese, E. Gurevich, F. Baboux, T. Jacqmin, A. Lemaître, E. Galopin, I. Sagnes, A. Amo, J. Bloch, and E. Akkermans, Phys. Rev. Lett. 112, 146404 (2014).

8 S. Barrett, K. Hammerer, S. Harrison, T. E. Northup, and T. J. Osborne, Phys. Rev. Lett. 110, 090501 (2013).

9 C. Eichler, J. Mlynek, J. Butscher, P. Kurpiers, K. Hammerer, T. J. Osborne, and A. Wallraff, Phys. Rev. X 5, 041044 (2015)

${ }^{10}$ R. Barends, L. Lamata, J. Kelly, L. García-Álvarez, A. G. Fowler, A. Megrant, E. Jeffrey, T. C. White, D. Sank, J. Y. Mutus, B. Campbell, Y. Chen, Z. Chen, B. Chiaro, A. Dunsworth, I. C. Hoi, C. Neill, P. J. J. O'Malley, C. Quintana, P. Roushan, A. Vainsencher, J. Wenner, E. Solano, and J. M. Martinis, Nat Comms 6, 7654 (2015).

11 I. Buluta and F. Nori, Science 326, 108 (2009).

12 T. Kadowaki and H. Nishimori, Phys. Rev. E 58, 5355 (1998).

13 Y. Zhang, L. Yu, J.-Q. Liang, G. Chen, S. Jia, and F. Nori, Sci. Rep. 4 (2014).

14 A. Blais, R.-S. Huang, A. Wallraff, S. M. Girvin, and R. J. Schoelkopf, Phys. Rev. A 69, 062320 (2004).
15 C. Lang, D. Bozyigit, C. Eichler, L. Steffen, J. Fink, A. Abdumalikov Jr, M. Baur, S. Filipp, M. da Silva, A. Blais, et al., Phys. Rev. Lett. 106, 243601 (2011).

16 A. J. Hoffman, S. J. Srinivasan, S. Schmidt, L. Spietz, J. Aumentado, H. E. Türeci, and A. A. Houck, Phys. Rev. Lett. 107, 053602 (2011).

17 X. Deng, C. Jia, and C.-C. Chien, Phys. Rev. B 91, 054515 (2015).

18 A. Baust, E. Hoffmann, M. Haeberlein, M. J. Schwarz, P. Eder, J. Goetz, F. Wulschner, E. Xie, L. Zhong, F. Quijandría, B. Peropadre, D. Zueco, J.-J. García Ripoll, E. Solano, K. Fedorov, E. P. Menzel, F. Deppe, A. Marx, and R. Gross, Phys. Rev. B 91, 014515 (2015).

19 R. Bialczak, M. Ansmann, M. Hofheinz, M. Lenander, E. Lucero, M. Neeley, A. OConnell, D. Sank, H. Wang, M. Weides, et al., Phys. Rev. Lett. 106, 060501 (2011).

${ }^{20}$ Y. Chen, C. Neill, P. Roushan, N. Leung, M. Fang, R. Barends, J. Kelly, B. Campbell, Z. Chen, B. Chiaro, et al., Phys. Rev. Lett. 113, 220502 (2014).

21 M. P. A. Fisher, P. B. Weichman, G. Grinstein, and D. S. Fisher, Phys. Rev. B 40, 546 (1989).

22 I. Bloch, J. Dalibard, and S. Nascimbène, Nat. Phys. 8, 267 (2012).

23 P. Buonsante, R. Burioni, D. Cassi, V. Penna, and A. Vezzani, Physical Review B 70, 224510 (2004).

24 R. Roth and K. Burnett, Physical Review A 68, 023604 (2003).

25 D. Jaksch, C. Bruder, J. I. Cirac, C. W. Gardiner, and P. Zoller, Physical Review Letters 81, 3108 (1998).

${ }^{26}$ G.-B. Jo, J. Guzman, C. K. Thomas, P. Hosur, A. Vishwanath, and D. M. Stamper-Kurn, Physical review letters 108, 045305 (2012). 
27 P. Buonsante, V. Penna, and A. Vezzani, Physical Review A 72, 031602 (2005).

${ }^{28}$ S. D. Huber and E. Altman, Phys. Rev. B 82, 184502 (2010).

29 E. J. Bergholtz and Z. Liu, Int. J. Mod Phys B 27, 1330017 (2013).

30 S. Flach, D. Leykam, J. D. Bodyfelt, P. Matthies, and A. S. Desyatnikov, Europhys. Lett. 105, 30001 (2014).

31 S. A. Parameswaran, R. Roy, and S. L. Sondhi, C R Phys 14, 816 (2013).

32 O. Derzhko, J. Richter, A. Honecker, M. Maksymenko, and R. Moessner, Phys. Rev. B 81, 014421 (2010).

33 A. Mielke, J. Phys. A 24, L73 (1991).

34 H. Tasaki, Phys. Rev. Lett. 69, 1608 (1992).

35 T. Zhang and G.-B. Jo, Sci. Rep. 5, 16044 (2015).

${ }^{36}$ I. Bloch, J. Dalibard, and W. Zwerger, Rev. Mod. Phys. 80, 885 (2008)

37 N. Masumoto, N. Y. Kim, T. Byrnes, K. Kusudo, A. Löffler, S. Höfling, A. Forchel, and Y. Yamamoto, New J. Phys. 14, 065002 (2012).

${ }^{38}$ D. N. Christodoulides, F. Lederer, and Y. Silberberg, Nature 424, 817 (2003).

39 S. Hacohen-Gourgy, V. V. Ramasesh, C. De Grandi, I. Siddiqi, and S. M. Girvin, Phys. Rev. Lett. 115, 240501 (2015).

40 Y. Makhlin, G. Schön, and A. Shnirman, Rev. Mod. Phys. 73, 357 (2001).

41 B. Peropadre, D. Zueco, F. Wulschner, F. Deppe, A. Marx, R. Gross, and J. J. García-Ripoll, Phys. Rev. B 87, 134504 (2013).

42 F. Wulschner, J. Goetz, F. Koessel, E. Hoffmann, A. Baust, P. Eder, M. Fischer, M. Haeberlein, M. Schwarz, M. Pernpeintner, et al., arXiv:1508.06758 (2015).

43 M. Leib, F. Deppe, A. Marx, R. Gross, and M. Hartmann, New Journal of Physics 14, 075024 (2012).

44 J. Koch and K. Le Hur, Phys. Rev. A 80, 023811 (2009).

45 K. Seo and L. Tian, Physical Review B 91, 195439 (2015).

46 M. Mariantoni, H. Wang, R. C. Bialczak, M. Lenander, E. Lucero, M. Neeley, A. OConnell, D. Sank, M. Weides, J. Wenner, et al., Nat. Phys. 7, 287 (2011).

47 H. Wang, M. Hofheinz, J. Wenner, M. Ansmann, R. Bialczak, M. Lenander, E. Lucero, M. Neeley, A. OConnell, D. Sank, et al., Appl. Phys. Lett. 95, 233508 (2009).

48 M. Reagor, H. Paik, G. Catelani, L. Sun, C. Axline, E. Holland, I. M. Pop, N. A. Masluk, T. Brecht, L. Frunzio, et al., Appl. Phys. Lett. 102, 192604 (2013).

49 N. Matsuda, H. Le Jeannic, H. Fukuda, T. Tsuchizawa, W. J. Munro, K. Shimizu, K. Yamada, Y. Tokura, and H. Takesue, Sci. Rep. 2 (2012).

50 D. L. Underwood, W. E. Shanks, J. Koch, and A. A. Houck, Phys. Rev. A 86, 023837 (2012).

51 A. Shcherbakova, K. Fedorov, K. Shulga, V. Ryazanov, V. Bolginov, V. Oboznov, S. Egorov, V. Shkolnikov, M. Wolf, D. Beckmann, et al., Supercond. Sci. Technol. 28, 025009 (2015).

${ }^{52}$ X. Deng, Y. Hu, and L. Tian, Supercond. Sci. Technol.
26, 114002 (2013).

53 J. Mooij, T. Orlando, L. Levitov, L. Tian, C. H. Van der Wal, and S. Lloyd, Science 285, 1036 (1999).

54 A. Wallraff, D. I. Schuster, A. Blais, L. Frunzio, R.-S. Huang, J. Majer, S. Kumar, S. M. Girvin, and R. J. Schoelkopf, Nature 431, 162 (2004).

55 T. Lanting, A. Berkley, B. Bumble, P. Bunyk, A. Fung, J. Johansson, A. Kaul, A. Kleinsasser, E. Ladizinsky, F. Maibaum, et al., Phys. Rev. B 79, 060509 (2009).

56 J. M. Zhang and R. X. Dong, Eur. J. Phys. 31, 591 (2010).

57 P. Buonsante, V. Penna, and A. Vezzani, Phys. Rev. A 82, 043615 (2010).

58 A. A. Gangat, I. A. McCulloch, and G. J. Milburn, Phys. Rev. X 3, 031009 (2013).

59 A. V. Sharypov, X. Deng, and L. Tian, Phys. Rev. B 86, 014516 (2012).

60 B. Johnson, M. Reed, A. Houck, D. Schuster, L. S. Bishop, E. Ginossar, J. Gambetta, L. DiCarlo, L. Frunzio, S. Girvin, et al., Nat. Phys. 6, 663 (2010).

61 C. Guerlin, J. Bernu, S. Deleglise, C. Sayrin, S. Gleyzes, S. Kuhr, M. Brune, J.-M. Raimond, and S. Haroche, Nature 448, 889 (2007).

62 D. Schuster, A. Houck, J. Schreier, A. Wallraff, J. Gambetta, A. Blais, L. Frunzio, J. Majer, B. Johnson, M. Devoret, et al., Nature 445, 515 (2007).

63 M. Mirrahimi, Z. Leghtas, V. V. Albert, S. Touzard, R. J. Schoelkopf, L. Jiang, and M. H. Devoret, New J. Phys. 16, 045014 (2014).

64 A. Sarlette, J.-M. Raimond, M. Brune, and P. Rouchon, Phys. Rev. Lett. 107, 010402 (2011).

65 A. Roy, Z. Leghtas, A. D. Stone, M. Devoret, and M. Mirrahimi, Phys. Rev. A 91, 013810 (2015).

66 C. Rigetti, J. M. Gambetta, S. Poletto, B. Plourde, J. M. Chow, A. Córcoles, J. A. Smolin, S. T. Merkel, J. Rozen, G. A. Keefe, et al., Phys. Rev. B 86, 100506 (2012).

67 Y. Makhlin, G. Schön, and A. Shnirman, Rev. Mod. Phys. 73, 357 (2001)

${ }^{68}$ D. Greif, T. Uehlinger, G. Jotzu, L. Tarruell, and T. Esslinger, Science 340, 1307 (2013).

69 U. Schneider, L. Hackermüller, J. P. Ronzheimer, S. Will, S. Braun, T. Best, I. Bloch, E. Demler, S. Mandt, D. Rasch, et al., Nature Physics 8, 213 (2012).

70 Y. Nakamura, Y. A. Pashkin, and J. Tsai, Nature 398, 786 (1999).

71 J. Clarke and F. K. Wilhelm, Nature 453, 1031 (2008).

72 A. Baust, E. Hoffmann, M. Haeberlein, M. J. Schwarz, P. Eder, J. Goetz, F. Wulschner, E. Xie, L. Zhong, F. Quijandria, D. Zueco, J. J. G. Ripoll, L. García-Álvarez, G. Romero, E. Solano, K. G. Fedorov, E. P. Menzel, F. Deppe, A. Marx, and R. Gross, arXiv:1412.7372 (2014).

73 J. Bourassa, F. Beaudoin, J. M. Gambetta, and A. Blais, Phys. Rev. A 86, 013814 (2012).

74 L. I. Schiff, Quantum Mechanics 3rd (New York: McGrawHill, 1968). 